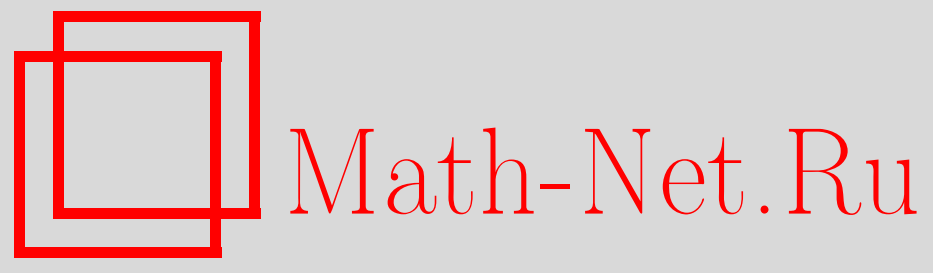

Д. С. Петухов, И. Э. Келлер, Двойственные задачи плоских ползущих течений степенной несжимаемой среды, Вестн. Сам. гос. техн. ун-та. Сер. Физ.-мат. науки, 2016, номер 3, 496-507

DOI: https://doi.org/10.14498/vsgtu1508

Использование Общероссийского математического портала MathNet.Ru подразумевает, что вы прочитали и согласны с пользовательским соглашением

http://www.mathnet.ru/rus/agreement

Параметры загрузки:

IP: 35.173 .219 .149

26 апреля 2023 г., 13:28:09

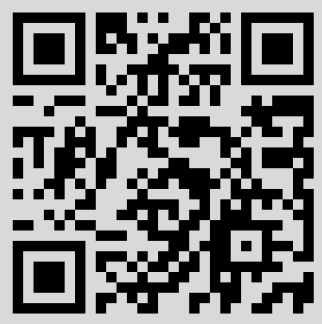


УДК 539.376

\title{
ДВОЙСТВЕННЫЕ ЗАДАЧИ ПЛОСКИХ ПОЛЗУЩИХ ТЕЧЕНИЙ СТЕПЕННОЙ НЕСЖИМАЕМОЙ СРЕДЫ
}

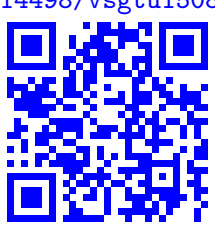

\section{Д. С. Петухов, И. Э. Келлер}

Институт механики сплошных сред УрО РАН, Россия, 614013, Пермь, ул. Акад. Королёва, 1.

\begin{abstract}
Аннотация
Для уравнений равновесия и совместности, описывающих ползущие плоские течения несжимаемой среды со степенной реологией, рассмотрен класс решений в форме произведения произвольной степени радиальной координаты на произвольную функцию угловой координаты полярной системы координат, покрывающей плоскость. Данный класс решений представляет асимптотику полей вблизи особой точки области, занятой рассматриваемой средой. Показана трансформация друг в друга точечными преобразованиями двух задач для плоскости с клиновидным вырезом, в одной из которых на границах выреза исчезают компоненты вектора поверхностных сил, а в другой - компоненты вектора скоростей. В ходе таких преобразований уравнения равновесия и совместности системы полевых уравнений переходят друг в друга, граничные условия одной задачи переходят в граничные условия другой задачи, а показатель степени реологического уравнения обращается. Для указанных двойственных нелинейных задач на собственные значения были изучены собственные решения и асимптотика полей вблизи вершины выреза в зависимости от показателя степени реологического уравнения и угла раствора выреза. При этом исследовалась ветвь собственных значений, связанная с собственным числом Хатчинсона-Райса-Розенгрена, известным по задаче о распределении напряжений в плоскости с разрезом для степенной среды. Двойственная задача дает распределение скоростей перемещений при течении степенной среды вблизи вершины жесткого клина. Найдены аналитические выражения для еще двух собственных чисел и установлено, что каждое из этих чисел отвечает за определенную простую структуру полей скоростей перемещений или напряжений в каждой из двойственных задач. Одно из этих собственных значений соответствует радиальному характеру течения среды и было обнаружено В. В. Соколовским, а в двойственной задаче отсутствует окружная компонента напряжений. Другое собственное значение соответствует одной ненулевой радиальной компоненте напряжений, а в двойственной задаче поле скоростей тривиально.
\end{abstract}

(C) 2016 Самарский государственный технический университет.

\section{Образец для цитирования}

Петухов Д. С., К еллер И. Э. Двойственные задачи плоских ползущих течений степенной несжимаемой среды // Вестн. Сам. гос. техн. ун-та. Сер. Физ.мат. науки, 2016. T. 20, № 3. С. 496-507. doi: 10.14498/vsgtu1508.

\section{Сведения об авторах}

Дмитрий Сергеевич Петухов (petuhovds@mail.ru), аспирант, лаб. нелинейной механики деформируемого твердого тела.

Илья Эрнстович Келлер (д.ф.-м.н., доц.; kie@icmm.ru; автор, ведущий переписку), научный сотрудник, лаб. нелинейной механики деформируемого твердого тела. 
Ключевые слова: установившаяся ползучесть, степенная реология, двойственность, разделение переменных, механика трещин, течения в сходящихся каналах.

Введение. В работе изучается класс инвариантных решений плоских уравнений равновесия несжимаемой среды со степенным реологическим законом, допускающий естественную запись в полярных координатах. Рассматриваемые уравнения в равной степени применимы для описания квазистатических течений вязкой степенной жидкости либо пластического твердого тела со степенным упрочнением вблизи геометрической особенности области. Подобные решения представляют ценность для определения расходно-напорных характеристик ползущих течений неньютоновских жидкостей в каналах, энергосиловых параметров процессов обработки металлов давлением [1-3], концентрации напряжений в задачах механики трещин в условиях ползучести [4-6], распределения полей вблизи вершины жесткого клина, обтекаемого ползущей средой либо внедряемого в ползущую среду со степенной реологией. Продвижение в понимании указанного выше класса решений и их приложений может быть достигнуто анализом решений некоторой нелинейной задачи на собственные значения.

Двойственные задачи в случае произвольной реологии. Рассматриваются плоские ползущие течения несжимаемой обобщенно-вязкой среды, подчиняющиеся обезразмеренным уравнениям:

$$
\begin{gathered}
\partial_{x} \sigma_{x x}+\partial_{y} \sigma_{x y}=0, \quad \partial_{x} \sigma_{x y}+\partial_{y} \sigma_{y y}=0, \quad \partial_{x} v_{x}+\partial_{y} v_{y}=0, \\
2 \sigma_{x x}+p=-2 \sigma_{y y}-p=\tau(\xi) \xi^{-1} \partial_{x} v_{x}, 4 \sigma_{x y}=\tau(\xi) \xi^{-1}\left(\partial_{y} v_{x}+\partial_{x} v_{y}\right), \\
\xi=\sqrt{\left(\partial_{x} v_{x}\right)^{2}+\frac{1}{4}\left(\partial_{y} v_{x}+\partial_{x} v_{y}\right)^{2}}
\end{gathered}
$$

где $\sigma_{x x}, \sigma_{y y}, \sigma_{x y}$ - компоненты тензора напряжений, $p=-\left(\sigma_{x x}+\sigma_{y y}\right)-$ удвоенное гидростатическое давление, $v_{x}$ и $v_{y}$ - компоненты вектора скорости, $x$ и $y$ - декартовы ортогональные координаты, а $\partial_{x}, \partial_{y}$ - частные производные по ним. Функция $\tau(\xi)$ в уравнениях (1) полагается обратимой, а в остальном произвольной. Из (1) следует, что

$$
\tau=\sqrt{\left(\sigma_{x x}-\sigma_{y y}\right)^{2}+4 \sigma_{x y}^{2}}
$$

а величина $\xi \tau$ равна скорости диссипации энергии.

Из уравнений (1) удобно исключить радикал. С этой целью для компонент тензоров напряжений и деформаций скорости записываются выражениями [7]:

$$
\begin{gathered}
2 \sigma_{x x}=-p-\tau \sin \phi, \quad 2 \sigma_{y y}=-p+\tau \sin \phi, \quad 2 \sigma_{x y}=\tau \cos \phi \\
\partial_{x} v_{x}=-\xi \sin \phi, \quad \partial_{y} v_{y}=\xi \sin \phi, \quad \partial_{y} v_{x}+\partial_{x} v_{y}=2 \xi \cos \phi
\end{gathered}
$$

гарантирующими пропорциональность этих тензоров и несжимаемость среды. Здесь $\phi$-удвоенный угол между одной из линий максимальных касательных напряжений и осью $x$. Зависимости (2) подставляются в уравнения 
равновесия, а соотношения (3) совместно с выражением для вихря скорости

$$
q=\frac{1}{2}\left(\partial_{x} v_{y}-\partial_{y} v_{x}\right)
$$

разрешаются в виде

$$
\partial_{x} v_{y}=q+\xi \cos \phi, \quad \partial_{y} v_{x}=-q+\xi \cos \phi
$$

и трансформируются с помощью соотношений

$$
\partial_{y}\left(\partial_{x} v_{x}\right)=\partial_{x}\left(\partial_{y} v_{x}\right), \quad \partial_{x}\left(\partial_{y} v_{y}\right)=\partial_{y}\left(\partial_{x} v_{y}\right)
$$

в уравнения совместности деформации скорости.

В итоге уравнения (1) с учетом (2)-(4) записываются в виде системы однородных квазилинейных уравнений первого порядка

$$
\begin{aligned}
& \partial_{x} p+\sin \phi \partial_{x} \tau+\tau \cos \phi \partial_{x} \phi-\cos \phi \partial_{y} \tau+\tau \sin \phi \partial_{y} \phi=0 \\
& \partial_{y} p-\cos \phi \partial_{x} \tau+\tau \sin \phi \partial_{x} \phi-\sin \phi \partial_{y} \tau-\tau \cos \phi \partial_{y} \phi=0 \\
& \partial_{x} q-\cos \phi \partial_{x} \xi+\xi \sin \phi \partial_{x} \phi-\sin \phi \partial_{y} \xi-\xi \cos \phi \partial_{y} \phi=0 \\
& \partial_{y} q-\sin \phi \partial_{x} \xi-\xi \cos \phi \partial_{x} \phi+\cos \phi \partial_{y} \xi-\xi \sin \phi \partial_{y} \phi=0
\end{aligned}
$$

Если граница $y=0$ свободна от усилий, то на ней должны выполняться соотношения

$$
2 \sigma_{y y}=-p+\tau \sin \phi=0, \quad 2 \sigma_{x y}=\tau \cos \phi=0 .
$$

Если же граница $y=0$ непроницаема для движения сквозь нее среды и на ней имеет место прилипание, то на ней должны выполняться соотношения

$$
\partial_{x} v_{y}=q+\xi \cos \phi=0, \quad \partial_{x} v_{x}=-\xi \sin \phi=0 .
$$

Система (5) инвариантна относительно точечного преобразования

$$
\xi=\bar{\tau}, \quad \tau=\bar{\xi}, \quad q=\bar{p}, \quad p=-\bar{q}, \quad \phi=\bar{\phi}-\pi / 2 .
$$

Данное преобразование трансформирует граничные условия (6) и (7) друг в друга. Это означает, что решение задачи (5), (6) (или (5), (7)) с реологической функцией $\tau(\xi)$ является решением задачи $(5),(7)$ (или соответственно $(5),(6))$ с реологической функцией, обратной к $\tau(\xi)$.

Если условия исчезновения поверхностной силы или исчезновения скоростей перемещений выполняются на луче $\varphi=\varphi_{*}$, записанном в терминах полярной системы координат $r, \varphi$, то равенства (6) и (7) принимают соответствующий вид:

$$
\begin{gathered}
2 \sigma_{\varphi \varphi}=-p+\tau \sin w=0, \quad 2 \sigma_{r \varphi}=\tau \cos w=0 \\
\partial_{r} v_{\varphi}=q+\xi \cos w=0, \quad \partial_{r} v_{r}=-\xi \sin w=0
\end{gathered}
$$

где $w=\phi-2 \varphi$. Установленное выше свойство взаимности граничных условий (8) и (9) остается в силе. 
Следует отметить, что задачи на основе полевых уравнений (5) с граничными условиями (6) либо (7) являются однородными.

Двойственные решения с разделяющимися переменными для степенной реологии. Будем полагать, что реологическая функция имеет степенной вид $\tau=\xi^{m}$. Значения показателя степени $m=0$ соответствуют идеально пластическому твердому телу, $m=1$ - линейно вязкой (ньютоновской) жидкости, между ними располагаются значения, соответствующие реологии некоторых жидкостей, полимеров, а также металлов в установившейся стадии ползучести при повышенных температурах.

Уравнения (5) с $\tau=\xi^{m}$ принимают вид

$\partial_{x} p+m \xi^{m-1} \sin \phi \partial_{x} \xi+\xi^{m} \cos \phi \partial_{x} \phi-m \xi^{m-1} \cos \phi \partial_{y} \xi+\xi^{m} \sin \phi \partial_{y} \phi=0$,

$\partial_{y} p-m \xi^{m-1} \cos \phi \partial_{x} \xi+\xi^{m} \sin \phi \partial_{x} \phi-m \xi^{m-1} \sin \phi \partial_{y} \xi-\xi^{m} \cos \phi \partial_{y} \phi=0$,

$\partial_{x} q-\cos \phi \partial_{x} \xi+\xi \sin \phi \partial_{x} \phi-\sin \phi \partial_{y} \xi-\xi \cos \phi \partial_{y} \phi=0$,

$\partial_{y} q-\sin \phi \partial_{x} \xi-\xi \cos \phi \partial_{x} \phi+\cos \phi \partial_{y} \xi-\xi \sin \phi \partial_{y} \phi=0$.

Система (10) инвариантна относительно точечного преобразования $\xi=a \bar{\xi}$, $q=a \bar{q}, p=a^{m} \bar{p}, \phi=\bar{\phi}$ с параметром $a>0$, а также поворотов и растяжений в плоскости независимых переменных. Произвольной комбинации таких преобразований соответствует инфинитезимальный оператор

$$
X=\beta r \partial_{r}+\alpha \partial_{\varphi}+2 \alpha \partial_{\phi}+\xi \partial_{\xi}+q \partial_{q}+m p \partial_{p},
$$

где $r, \varphi$ - полярные координаты; $\alpha, \beta$ - произвольные параметры. Оператор (11) при заданных $\alpha, \beta$ генерирует определенную подалгебру - элемент оптимальной системы одномерных подалгебр алгебры Ли инфинитезимальных операторов преобразований, допускаемых системой (10) [8-10].

Для случая $\beta \neq 0$ набор базисных инвариантов преобразования с инфинитезимальным оператором (11) выглядит следующим образом:

$$
z=\frac{\alpha}{\beta} \ln r-\varphi, \quad f=r^{-1 / \beta} \xi, \quad h=r^{-1 / \beta} q, \quad g=r^{-m / \beta} p, \quad w=\phi-2 \varphi .
$$

В настоящей работе ограничимся случаем $\alpha=0$, отвечающим за разделение радиальной и угловой независимых переменных. Неособое инвариантное решение

$$
\xi=r^{1 / \beta} f(z), \quad q=r^{1 / \beta} h(z), \quad p=r^{m / \beta} g(z), \quad w=w(z), \quad z=-\varphi
$$

удовлетворяет системе обыкновенных дифференциальных уравнений (факторсистеме)

$$
\begin{aligned}
& \beta f^{m} w^{\prime}-(2 \beta+m) f^{m}-\beta g^{\prime} \cos w-m g \sin w=0, \\
& \beta m f^{m-1} f^{\prime}+m g \cos w-\beta g^{\prime} \sin w=0, \\
& \beta f w^{\prime}-(2 \beta+1) f+h \cos w-\beta h^{\prime} \sin w=0, \\
& \beta f^{\prime}+\beta h^{\prime} \cos w+h \sin w=0 .
\end{aligned}
$$

Данная система преобразуется к паре уравнений для функций $f, w$ :

$$
\begin{aligned}
& \frac{\beta^{2}}{m^{2}}\left(f^{m} \cos w\right)^{\prime \prime}+2 \frac{\beta}{m}\left(\frac{\beta}{m}+1\right)\left(f^{m} \sin w\right)^{\prime}-\left(2 \frac{\beta}{m}+1\right) f^{m} \cos w=0, \\
& \beta^{2}(f \sin w)^{\prime \prime}-2 \beta(\beta+1)(f \cos w)^{\prime}-(2 \beta+1) f \sin w=0
\end{aligned}
$$


и выражениям для функций $g, h$ :

$$
\begin{aligned}
g & =\frac{\beta}{m}\left(f^{m} \cos w\right)^{\prime}+\left(2 \frac{\beta}{m}+1\right) f^{m} \sin w, \\
h & =\beta(f \sin w)^{\prime}-(2 \beta+1) f \cos w .
\end{aligned}
$$

Для численного решения удобна запись системы (12) в нормальной форме Коши:

$$
\begin{aligned}
& f^{\prime}=\Delta^{-1}\left((1-m) f \sin w \cos w-m f^{1-m} g \cos w-h \sin w\right), \\
& w^{\prime}=\Delta^{-1} m\left(f^{-1} \cos w-f^{-m} g \sin w\right)-\left(2+\beta^{-1} m\right), \\
& g^{\prime}=m(1-m)\left(f^{m} \cos w+g \sin w\right)-\frac{m}{2} f^{m-1} g, \\
& h^{\prime}=(1-m)(f-h \cos w) \sin w-m f^{1-m} g,
\end{aligned}
$$

где $\Delta=\beta\left(m \cos ^{2} w+\sin ^{2} w\right) \neq 0$.

Граничные условия (8) и (9) в рамках рассматриваемого класса решений с учетом $z= \pm \varphi_{*},\left|\varphi_{*}\right|<\pi$ примут соответственно вид

$$
g-f^{m} \sin w=0, \quad f^{m} \cos w=0 .
$$

И

$$
h+f \cos w=0, \quad f \sin w=0 .
$$

Можно убедиться, что система (13), (14) инвариантна при точечном преобразовании

$$
f=\bar{f}^{\bar{m}}, \quad h=\bar{g}, \quad g=-\bar{h}, \quad w=\bar{w}-\pi / 2, \quad m=1 / \bar{m}, \quad \beta=\bar{\beta} / \bar{m},
$$

а граничные условия (16), (17) при этом переходят друг в друга. Система (15) также является инвариантной относительно указанных преобразований. Поэтому решение задачи (13), (14) при определенном значении $m$ и граничных условиях (16) (или (17)) на $z= \pm \varphi_{*}$ посредством (18) преобразуется в решение задачи, соответствующей граничным условиям (17) (или (16)) и значению показателя степени реологического уравнения, равному $1 / \mathrm{m}$.

Задача об асимптотическом распределении напряжений у вершины выреза. Уравнения (15) с граничными условиями (16) при $\varphi_{*}=\pi$ представляют собой известную задачу об асимптотическом распределении напряжений у кончика трещины (разреза) в рамках плоского деформированного состояния. Строгие результаты о разрешимости этой задачи отсутствуют, однако одно собственное значение $\beta=-(m+1)$ (специализация Хатчинсона-Райса-Розенгрена) установлено в $[4,5]$.

Здесь данная задача решалась методом Рунге-Кутты в сочетании с методом пристрелки, ${ }^{1}$ для чего решалась начальная задача с условиями (16) в точке $z=-\pi$, условием нормировки $f(-\pi)=1$ и условием $h(-\pi)=\lambda$ для значений параметров $\lambda \in[-10,10]$ и $\beta$ из окрестности точки $-(m+1)$. Отрезок изменения $\lambda$ разбивался на 20000 точек. Было установлено, что нулевые значения невязки достигаются в некоторых точках сечения $\beta=-(m+1)$ заданной области параметров.

\footnotetext{
${ }^{1}$ Использовались стандартные функции пакета Wolfram Mathematica 10.
} 
На рис. 1 приведена невязка

$$
\delta=r^{-m / \beta} \sqrt{\sigma_{\varphi \varphi}^{2}+\sigma_{r \varphi}^{2}}
$$

граничных условий (16) в точке $z=\pi$ как функция параметра $\lambda$ для $m=0.2$. Нули невязки позволяют установить два собственных решения: с симметричными и антисимметричными угловыми распределениями компонент напряжений $\sigma_{r r}$ и $\sigma_{\varphi \varphi}$ относительно линии разреза (и соответственно антисимметричным и симметричным распределениями компоненты $\sigma_{r \varphi}$ ) (рис. 2). Первый случай условно назовем «нормальной», а второй - «сдвиговой» собственными формами. Этот результат воспроизводится для любого показателя степени из интервала $0<m<1$; это означает, что ни при каких нагружениях тела со степенной реологией $0<m<1$ напряженное состояние вблизи кончика трещины не может иметь смешанный характер и не соответствует общепринятой точке зрения [11-14].

Зависимость невязки от $\lambda$ для $m=5$ (рис. 3) обнаруживает четыре решения, два из которых соответствуют нормальной и сдвиговой собственным формам, а два оставшиеся - смешанной форме (рис. 4) и ее инверсии.

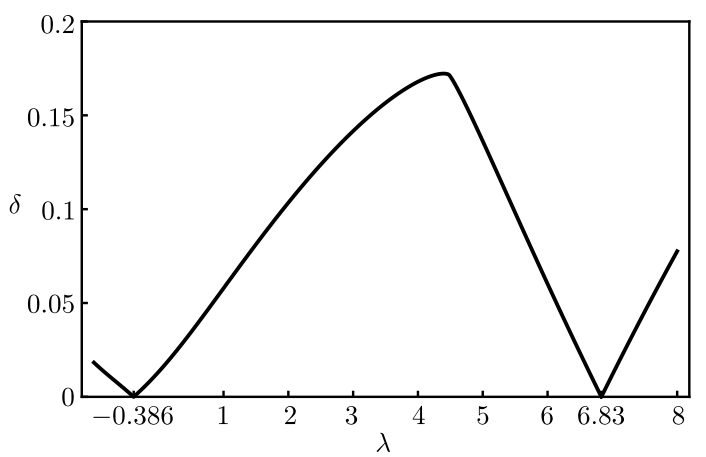

Рис. 1. Зависимость невязки граничных условий от параметра $\lambda$ в сечении $\beta=-(m+1)$ для $m=0.2$ [Figure 1. The dependence of the discrepancy of the boundary conditions from the parameter $\lambda$ in section $\beta=-(m+1)$ for $m=0.2$ ]
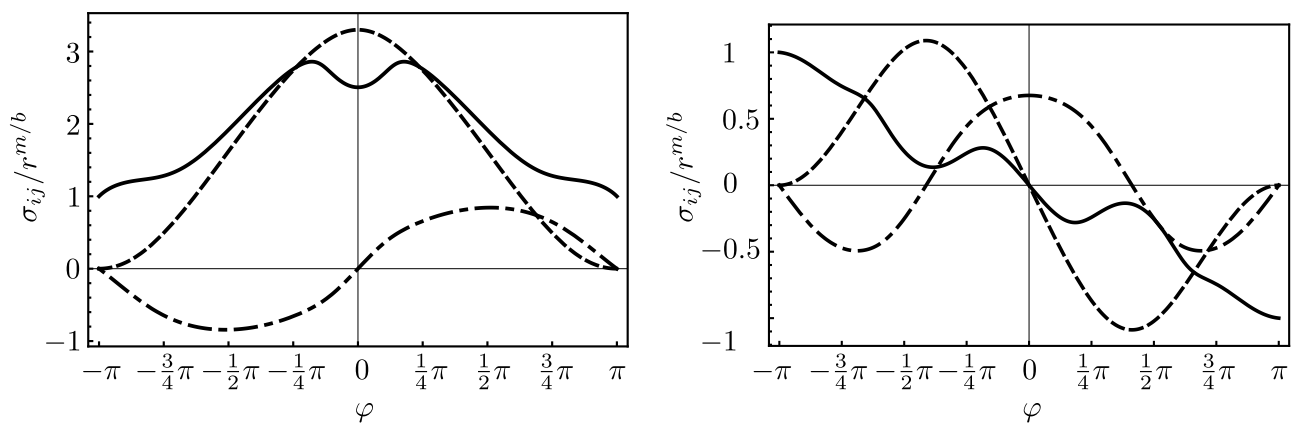

Рис. 2. Распределения напряжений для нормальной (слева) и сдвиговой (справа) собственных форм при $m=0.2$. Сплошная линия - радиальное, пунктирная - окружное, штрихпунктирная - сдвиговое напряжения. Специализация Хатчинсона-Райса-Розенгрена

[Figure 2. Stress distributions for the normal (left) and shear (right) eigenforms at $m=0.2$. Solid line is radial normal stress, dashed one is circumferential normal stress, dot-dashed one is shear stress. The Hutchinson-Rice-Rosengren specialization] 
Этот результат воспроизводится для любого показателя степени из интервала $m>1$.

На рис. 5 представлены зависимости показателя степени радиальной координаты в компонентах напряжений от угла раствора $\varphi_{*}$, ограничивающего вырез, для нормальной и сдвиговой собственных форм и $m=0.2$. На рис. 6 помещены аналогичные зависимости для $m=5$; смешанные собственные формы для $\varphi_{*} \neq \pi$ не найдены. Отметим, что для $\varphi_{*} \neq \pi$ также $\beta \neq-(m+1)$. Стоит обратить внимание на то, что при увеличении угла выреза асимптотика напряжений у его вершины перестает быть сингулярной для сдвиго-

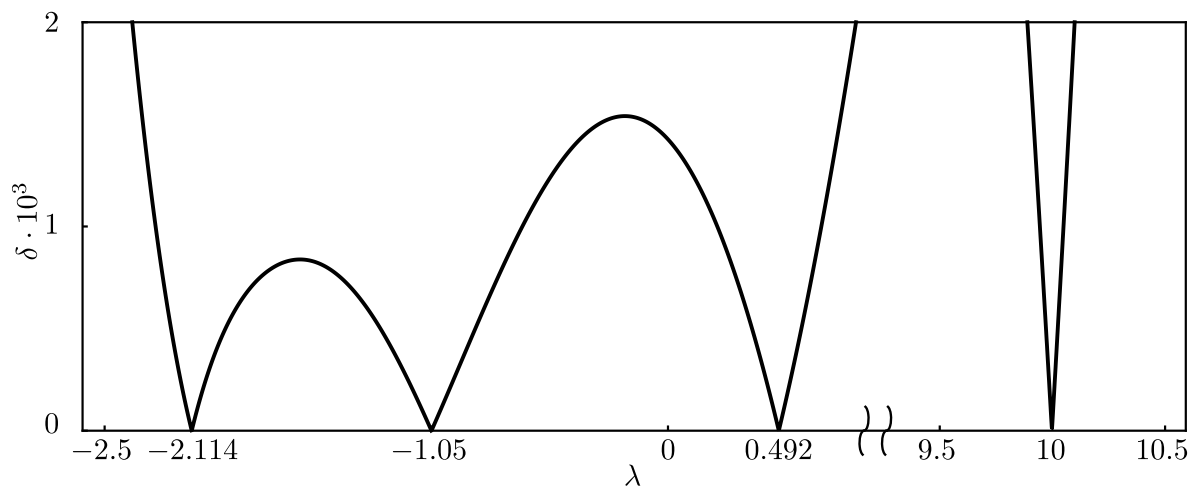

Рис. 3. Зависимость невязки граничных условий от параметра $\lambda$ в сечении $\beta=-(m+1)$ для $m=5$ [Figure 3. The dependence of the discrepancy of the boundary conditions from the parameter $\lambda$ in section $\beta=-(m+1)$ for $m=5$ ]
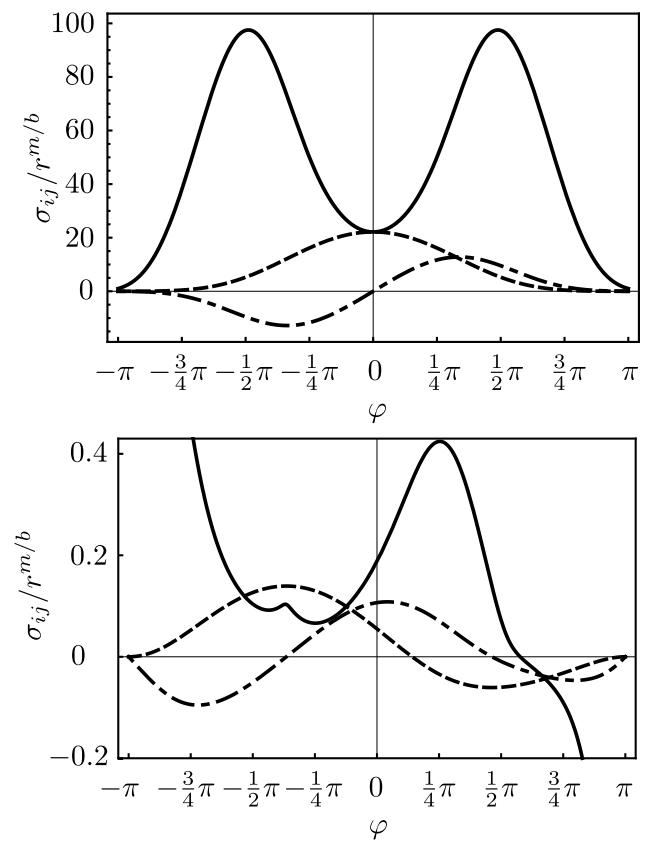

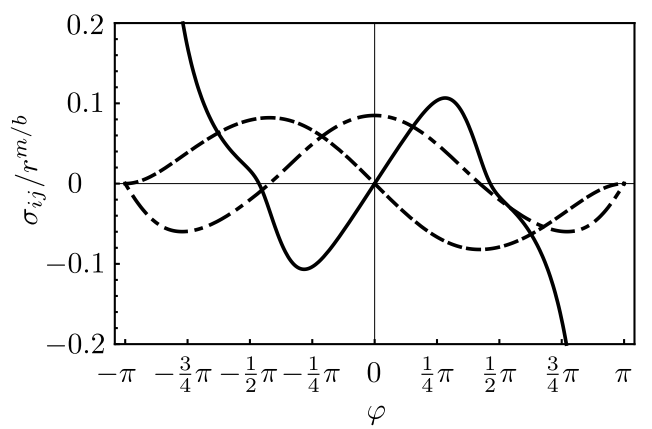

Рис. 4. Распределения напряжений для нормальной (слева), сдвиговой (справа) и смешанной (снизу) собственных форм при $m=5$. Сплошная линия-радиальное, пунктирная - окружное, штрихпунктирная - сдвиговое напряжения. Специализация Хатчинсона-Райса-Розенгрена

[Figure 4. Stress distribution for the normal (left), shear (right) и смешанной (bottom) eigenforms at $m=5$. Solid line is radial normal stress, dashed one is circumferential normal

stress, dot-dashed one is shear stress. The Hutchinson-Rice-Rosengren specialization] 

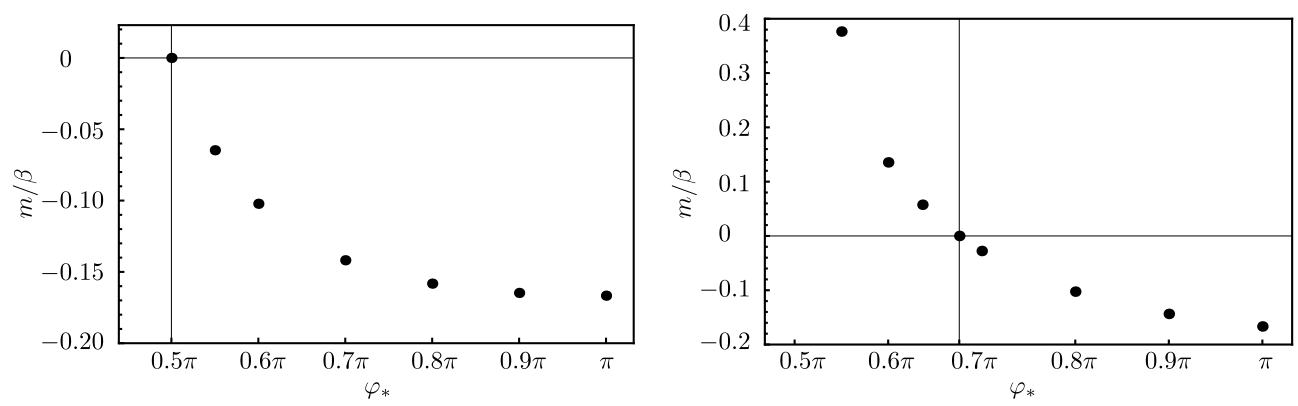

Рис. 5. Зависимость показателя степени радиальной координаты в компонентах напряжений от угла выреза для $m=0.2$. Слева - нормальная, справа - сдвиговая собственные формы. Специализация Хатчинсона-Райса-Розенгрена

[Figure 5. The dependence of the power law exponent of the radial coordinate in stress components from the angle of the cut for $m=0.2$. The normal eigenform on the left side and

the shear eigenform on the right side. The Hutchinson-Rice-Rosengren specialization]
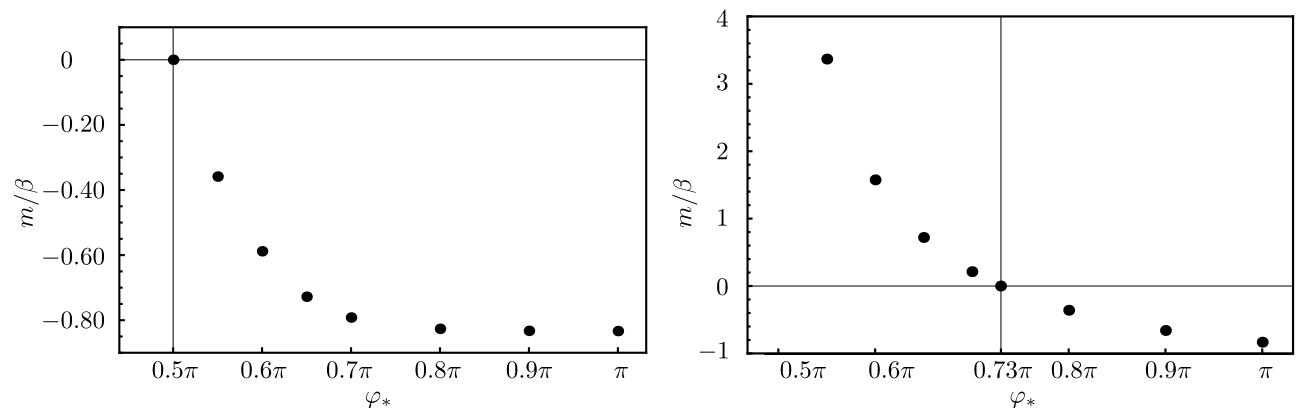

Рис. 6. Зависимость показателя степени радиальной координаты в компонентах напряжений от угла выреза для $m=5$. Слева - нормальная, справа-сдвиговая собственные формы. Специализация Хатчинсона-Райса-Розенгрена

[Figure 6. The dependence of the power law exponent of the radial coordinate in stress components from the angle of the cut for $m=5$. The normal eigenform on the left side and

the shear eigenform on the right side. The Hutchinson-Rice-Rosengren specialization]

вой собственной формы, оставаясь сингулярной для нормальной собственной формы. Для $\varphi_{*}<\pi / 2$ подобные решения не найдены.

Полученные выше результаты после известной трансформации могут представлять решение двойственной задачи о распределении скоростей вблизи вершины жесткого клина с условиями исчезновения поля скоростей перемещений на границах. Из рис. 5, 6 можно увидеть, что асимптотика компонент скоростей, пропорциональных $r^{\gamma}$, при $r \rightarrow 0$ для двойственной задачи не имеет сингулярности в вершине угла, так как $\gamma=m / b+1=1 /(m+1)>0$. Это необходимо принимать во внимание при интерпретации решений, отвечающих нормальной, сдвиговой и смешанной собственным формам задачи в скоростях.

О задачах, соответствующих некоторым другим собственным значениям. Численный эксперимент для задачи с клиновидным вырезом со свободными границами и произвольным $m$ выявил собственное число $\beta=-m / 2$. Несложно показать аналитически, что этот случай соответствует исчезновению поля тангенциальной компоненты тензора напряжений: $\sigma_{\varphi \varphi} \equiv 0$. При этом перво- 
му уравнению в системе (13) (уравнению равновесия) можно удовлетворить подстановкой $f^{m} \sin w=u^{\prime}(z), f^{m} \cos w=2 u(z)$.

Двойственной задачей к рассмотренной является задача о распределении скоростей вблизи вершины жесткого клина, на границах которого исчезают компоненты вектора скорости, соответствующая специализации $\beta=-1 / 2$. Двойственность данной задачи ведет к отсутствию тангенциальной компоненты вектора скорости $\left(v_{\varphi} \equiv 0\right)$ и следующему представлению радиальной компоненты вектора скорости:

$$
v_{r}=v(z) / r
$$

В этом случае второе уравнение системы (13) удовлетворяется тождественно при $f \cos w=0.5 v^{\prime}(z), f \sin w=v(z)$. Данный класс решений, исследованный В. В. Соколовским $[1,2]$, в отличие от специализации $\beta=-(m+1)$, имеет сингулярность (источник или сток) в начале системы координат и потому может быть интерпретирован как течение в сходящемся канале. Анализ спектра собственных форм (течений) в зависимости от угла раствора и $m$ будет опубликован отдельно.

Численные эксперименты для задачи со свободными границами разреза выявили еще ряд собственных значений. Одно из таких значений $\beta=-m$ соответствует исчезновению полей тангенциальной $\sigma_{\varphi \varphi}$ и сдвиговой $\sigma_{r \varphi}$ компонент тензора напряжений. Анализ спектра рассматриваемых задач представляется предметом отдельного исследования.

Заключение. Степенная реология среды при $m \neq 0$ и $m \neq 1$ допускает существование инвариантных решений уравнений равновесия и совместности (в частности, в рамках условий несжимаемости и плоской деформации), которые не очень хорошо изучены. Определенным препятствием для этого служит статическая и кинематическая неопределимость практически любой краевой задачи на базе данных уравнений в сочетании с радикалом, вносящим существенную техническую сложность в уравнения. Авторам известно немного работ (в частности [15]), в которых из этого факта была бы извлечена польза для более глубокого понимания механики движения таких сред. Основным результатом представленной работы является обнаружение связи двух задач на собственные значения, одна из которых естественным образом записывается в функциях, непосредственно связанных с напряжениями, а другаяв функциях, непосредственно связанных со скоростями перемещений. Оба класса задач имеют практическое применение и известны в литературе в областях нелинейной механики трещин и ползущих течений нелинейных сред в сходящихся каналах. Точечные преобразования (18), трансформирующие одну из задач и ее решения в двойственные, действуют иначе, чем точечные преобразования, обнаруженные в работе [16] для системы (5) с другой реологией $\tau(\xi)$. Результаты, представленные в работе, несколько расширяют известный класс течений В. В. Соколовского, характеризующийся очень ограниченной кинематикой движения среды и ее асимптотикой вблизи вершины угла или канала. Связь двух задач позволила найти пару новых собственных значений для механики трещин в дополнение к собственному значению Хатчинсона-Райса-Розенгрена, что позволяет надеяться на скорое решение проблемы разрешимости задачи механики трещин в теле со степенными определяющими соотношениями. 
Декларация о финансовых и других взаимоотношениях. Работа выполнена при поддержке программы УрО РАН (проект № 15-10-1-18). Все авторы принимали участие в разработке концепции статьи и в написании рукописи. Авторы несут полную ответственность за предоставление окончательной рукописи в печать. Окончательная версия рукописи была одобрена всеми авторами. Авторы не получали гонорар за статью.

\section{ORCIDs \\ Дмитрий Сергеевич Петухов: http://orcid.org/0000-0002-6996-3580 \\ Илья Эрнстович Келлер: http://orcid.org/0000-0001-9914-8870}

\section{БИБЛИОГРАФИЧЕСКИЙ СПИСОК}

1. Соколовский В. В. Плоское и осесимметричное равновесие пластической массы между жесткими стенками // ПММ, 1950. Т. 14, №1. С. 75-92.

2. Соколовский В. В. Теория пластичности. М.: Высш. шк., 1969. 608 с.

3. Малинин Н. Н. Технологические задачи пластичности и ползучести. М.: Высш. шк., 1979. $119 \mathrm{c}$.

4. Rice J. R., Rosengren G. F. Plane strain deformation near a crack tip in a power-law hardening material// J. Mech. Phys. Solids, 1968. vol.16, no.1. pp. 1-12. doi: 10.1016/ 0022-5096 (68) 90013-6.

5. Hutchinson J. W. Singular behaviour at the end of a tensile crack in a hardening material // J. Mech. Phys. Solids, 1968. vol. 16, no. 1. pp. 13-31. doi: 10.1016/0022-5096(68)90014-8.

6. Hutchinson J. W. Plastic stress and strain fields at a crack tip // J. Mech. Phys. Solids, 1968. vol. 16, no. 5. pp. 337-342. doi : 10.1016/0022-5096(68)90021-5.

7. Фрейденталь А., Гейрингер Х. Математические теории неупругой сплошной среды. М.: Физ.-мат. лит., 1962. 432 с.

8. Овсянников Л. В. Групповой анализ дифференциальных уравнений. М.: Наука, 1978. $400 \mathrm{c}$.

9. Головин С. В., Чесноков А. А. Групповой анализ дифференииальных уравнений. Новосибирск: Новосибирск. ун-т, 2008. 113 с.

10. Bluman G. W., Cheviakov A. F., Anco S. C. Applications of symmetry methods to partial differential equations / Applied Mathematical Sciences. vol. 168. Berlin, Heidelberg: SpringerVerlag, 2010. 414 pp. doi: 10.1007/978-0-387-68028-6.

11. Shih C. F. Elastic-plastic analysis of combined mode crack problems: Ph. D. Thesis. Cambridge, M.A.: Harvard University, 1973.

12. Shlyannikov V. N. Elastic-plastic mixed-mode fracture criteria and parameters/ Lecture Notes in Applied and Computational Mechanics. vol. 7. Berlin, Heidelberg: Springer-Verlag, 2003. 234 pp. doi : 10.1007/978-3-540-45836-4.

13. Астафьев В. И., Крутов А. Н. Распределение напряжений вблизи вершины наклонной трещины в нелинейной механике разрушения // Изв. РАН. МTT, 2001. № 5. С. 125-133.

14. Степанова Л.В., Яковлева Е. М. О смешанном нагружении элементов конструкции с дефектом // Вестн. Сам. гос. техн. ун-та. Сер. Физ.-мат. науки, 2015. Т. 19, № 2. C. 358-381. doi: 10.14498/vsgtu1432.

15. Душин В. Р. Инвариантные решения уравнений движения «степенных» жидкостей // Вестник Московского университета. Сер. 1, Математика, механика, 1988. № 2. С. 9195.

16. Келлер И. Э. Интегрируемость уравнений равновесия и совместности вязкопластической среды с отрицательной чувствительностью к скорости деформации // Докл. PAH, 2013. T. 451, № 6. C. 643-646.

Поступила в редакцию 13/VII/2016; в окончательном варианте - 26/VIII/2016; принята в печать - 09/IX/2016. 
Vestn. Samar. Gos. Techn. Un-ta. Ser. Fiz.-mat. nauki

[J. Samara State Tech. Univ., Ser. Phys. \& Math. Sci.], 2016, vol. 20, no. 3, pp. 496-507

ISSN: 2310-7081 (online), 1991-8615 (print)

doi: http://dx.doi.org/10.14498/vsgtu1508

MSC: 74D10, 74G55

\title{
DUAL PLANE PROBLEMS FOR CREEPING FLOW OF POWER-LAW INCOMPRESSIBLE MEDIUM
}

\author{
D. S. Petukhov, I. E. Keller
}

Institute of Continuous Media Mechanics, Ural Branch of RAS, 1, Akad. Korolyova st., Perm, 614013, Russian Federation.

\begin{abstract}
In this paper, we consider the class of solutions for a creeping plane flow of incompressible medium with power-law rheology, which are written in the form of the product of arbitrary power of the radial coordinate by arbitrary function of the angular coordinate of the polar coordinate system covering the plane. This class of solutions represents the asymptotics of fields in the vicinity of singular points in the domain occupied by the examined medium. We have ascertained the duality of two problems for a plane with wedge-shaped notch, at which boundaries in one of the problems the vector components of the surface force vanish, while in the other - the vanishing components are the vector components of velocity, We have investigated the asymptotics and eigensolutions of the dual nonlinear eigenvalue problems in relation to the rheological exponent and opening angle of the notch for the branch associated with the eigenvalue of the Hutchinson-Rice-Rosengren problem learned from the problem of stress distribution over a notched plane for a power law medium. In the context of the dual problem we have determined the velocity distribution in the flow of power-law medium at the vertex of a rigid wedge, We have also found another two eigenvalues, one of which was determined by V. V. Sokolovsky for the problem of power-law fluid flow in a convergent channel.
\end{abstract}

Keywords: steady-state creep, power-law rheology, duality, variable separation, crack mechanics, flow in convergent channel.

Declaration of Financial and Other Relationships. This work was supported by the program of the Ural Branch of RAS (project no. 15-10-1-18). Each author has participated in the article concept development and in the manuscript writing. The authors are absolutely responsible for submitting the final manuscript in print. Each author has approved the final version of manuscript. The authors have not received any fee for the article.

(C) 2016 Samara State Technical University.

Please cite this article in press as:

Petukhov D. S., Keller I. E. Dual plane problems for creeping flow of power-law incompressible medium, Vestn. Samar. Gos. Tekhn. Univ., Ser. Fiz.-Mat. Nauki [J. Samara State Tech. Univ., Ser. Phys. \& Math. Sci.], 2016, vol. 20, no. 3, pp. 496-507. doi: 10.14498/vsgtu1508. (In Russian)

\section{Authors Details:}

Dmitriy S. Petukhov (petuhovds@mail.ru), Postgraduate Student, Lab. of Nonlinear Mechanics of Deformable Solids.

Ilya E. Keller (Dr. Phys. \& Math. Sci.; kie@icmm.ru; Corresponding Author), Researcher, Lab. of Nonlinear Mechanics of Deformable Solids. 


\section{ORCIDs}

Dmitriy S. Petukhov: http://orcid.org/0000-0002-6996-3580

Ilya E. Keller: http://orcid.org/0000-0001-9914-8870

\section{REFERENCES}

1. Sokolovsky V. V. Plane and axisymmetric equilibrium of plastic mass between rigid walls, Prikl. Mat. Mekh., 1950, vol. 14, no. 1, pp. 75-92 (In Russian).

2. Sokolovsky V. V. Teoriia plastichnosti [Theory of Plasticity]. Moscow, Vysshaya Shkola Publ., 1969, 608 pp. (In Russian)

3. Malinin N. N. Tekhnologicheskie zadachi plastichnosti i polzuchesti [Technological problems of plasticity and creep]. Moscow, Vysshaya Shkola Publ., 1979, 119 pp. (In Russian)

4. Rice J. R., Rosengren G. F. Plane strain deformation near a crack tip in a power-law hardening material, J. Mech. Phys. Solids, 1968, vol.16, no.1, pp. 1-12. doi:10.1016/ 0022-5096 (68) 90013-6.

5. Hutchinson J. W. Singular behaviour at the end of a tensile crack in a hardening material, J. Mech. Phys. Solids, 1968, vol. 16, no. 1, pp. 13-31. doi: 10.1016/0022-5096(68)90014-8.

6. Hutchinson J. W. Plastic stress and strain fields at a crack tip, J. Mech. Phys. Solids, 1968, vol. 16, no. 5, pp. 337-342. doi: 10.1016/0022-5096(68)90021-5.

7. Freudental A. M., Geiringer H. The Mathematical Theories of the Inelastic Continuum, Handbuch der Physik, Bd. 6, Elasizitaet und Plastizitaet; ed. S. Flugge. Berlin, Goettingen, Heidelberg, Springer-Verlag, 1958, pp. 229-433. doi : 10.1007/978-3-642-45887-3_3.

8. Ovsyannikov L. V. Gruppovoi analiz differentsial'nykh uravnenii [Group analysis of differential equations]. Moscow, Nauka, 1978, 399 pp. (In Russian)

9. Golovin S. V., Chesnokov A. A. Gruppovoi analiz differentsial'nykh uravnenii [Group analysis of differential equations]. Novosibirsk, Novosibirsk State Univ., 2008, 113 pp. (In Russian)

10. Bluman G. W., Cheviakov A. F., Anco S. C. Applications of symmetry methods to partial differential equations, Applied Mathematical Sciences, vol. 168. Berlin, Heidelberg, SpringerVerlag, 2010, 414 pp. doi : 10.1007/978-0-387-68028-6.

11. Shih C. F. Elastic-plastic analysis of combined mode crack problems, Ph. D. Thesis. Cambridge, M.A., Harvard University, 1973.

12. Shlyannikov V. N. Elastic-plastic mixed-mode fracture criteria and parameters, Lecture Notes in Applied and Computational Mechanics, vol. 7. Berlin, Heidelberg, Springer-Verlag, 2003, 234 pp. doi : 10.1007/978-3-540-45836-4.

13. Astaf'ev V. I., Krutov A. N. Stress distribution near the tip of an inclined crack in the nonlinear fracture mechanics, Mech. Solids, 2001, vol. 36, no. 5, pp. 101-108.

14. Stepanova L. V., Yakovleva E. M. Mixed-mode loading of the structural elements with defect, Vestn. Samar. Gos. Tekhn. Univ., Ser. Fiz.-Mat. Nauki [J. Samara State Tech. Univ., Ser. Phys. \& Math. Sci.], 2015, vol.19, no. 2, pp. 358-381 (In Russian). doi: 10. 14498 /vsgtu1432.

15. Duchin V. R. Invariant solution of motion equations for "power-law" fluids, Vestn. Mosk. Univ., Ser. I. Matematika. Mekhanika, 1988, no. 2, pp. 91-95 (In Russian).

16. Keller I. E. Integrability of the Equilibrium and Compatibility Equations for a Viscoplastic Medium with Negative Strain Rate Sensitivity, Dokl. Phys., 2013, vol. 58, no. 8, pp. 643-646. doi : 10.1134/S1028335813080132.

Received 13/VII/2016; received in revised form $26 / \mathrm{VIII} / 2016$; accepted 09/IX/2016. 